\title{
Prevalência de Violência, Relação com Apoio Social e Sintomas Depressivos em Idosos
}

\author{
Prevalence of Violence, Relation with Social Support and Depressive \\ in the Elderly
}

Prevalencia de Violencia, Relación con Apoyo Social y Depresivos en Ancianos

Rodrigo da Silva Maia ${ }^{1}$

Eulália Maria Chaves Maia ${ }^{2}$

\section{Resumo}

Objetivo: caracterizar a prevalência de violência contra idosos residentes na região metropolitana do município de Natal, RN, e verificar a relação deste fenômeno com o apoio social e sintomas depressivos. Método: Estudo descritivo e transversal, realizado no período de 24 de outubro de 2016 a 26 de maio de 2017, com amostra, por conveniência, de 199 idosos residentes na comunidade. Os dados foram coletados por meio de: a) Questionário
Sócio Demográfico, b) a Escala de Screening da Vulnerabilidade ao Abuso (VASS-Br, c)a Escala de Depressão Geriátrica(GDS-15), e d) a Escala de Apoio Social (EAS). Resultados: Os dados obtidos foram categorizados e analisados através de estatística descritiva e inferencial. A maioria dos participantes foram mulheres $(n=141$, $70,9 \%$ ) com idade variando de 60 a 84 anos (M: 67,96; DP: $\pm 6,45$ ) residentes com cerca de uma a oito pessoas no domicílio (M: 4,07; DP: $\pm 1,36$ ). A prevalência de violência entre os idosos

${ }^{1}$ Psicólogo. Doutor pelo Programa de Pós-Graduação em Psicologia da UFRN. Docente do Centro Universitário UniFacex. Endereço completo: Rua Itamarati de Minas. No 2982, Apto 202. Neópolis. 59088-120. Natal-RN, Brasil. E-mail: rodrigo_maia89@yahoo.com.br

${ }^{2}$ Psicóloga. Doutora pelo Programa de Pós-Graduação em Psicologia Clínica da USP. Docente do Departamento de Psicologia da UFRN.

Recebido: Ago./2018 - Aceito: Dez./2018. 
da amostra foi de $16 \%(\mathrm{n}=31)$. Conclusão: Os achados apontam para prevalência de violência e associação com sintomas depressivos semelhantes a dados já citados na literatura. No entanto, ressalta-se que tais dados podem estar subnotificados, devido a aspectos como o vínculo de proximidade e/ou parentesco do agressor com a vítima ou as relações de dependência que existam na relação vítima-agressor.

\section{Descritores: Maus-Tratos ao Idoso;} Envelhecimento; Prevalência;

Depressão; Apoio Social.

\section{Abstract}

Objective: study was to characterize the prevalence of violence against elderly residents in the metropolitan region of Natal, Brazil, and to verify the relationship of this phenomenon with social support and depressive symptoms. Method: A descriptive and cross-sectional study, carried out from October 24, 2016 to May 26, 2017, with a sample, for convenience, of 199 elderly people living in the community. Data were collected by means of: a) Socio-demographic Questionnaire, b) the Abuse Vulnerability Screening Scale $(V A S S-B r, c)$ the Geriatric Depression
Scale (GDS-15), and d) the Social Support Scale AND THE). Results: The obtained data were categorized and analyzed through descriptive and inferential statistics. Most of the participants were women $(n=141$, $70.9 \%$ ), ranging in age from 60 to 84 years (M: 67.96; SD: \pm 6.45) with approximately one to eight persons at home (M: 4.07, DP: \pm 1.36). The prevalence of violence among the elderly in the sample was $16 \%(n=31)$. Conclusion: The findings point to the prevalence of violence and association with depressive symptoms similar to data already mentioned in the literature. However, it is emphasized that such data may be under-reported due to aspects such as the proximity bond and / or kinship of the aggressor with the victim or the dependency relations that exist in the victimaggressor relationship.

\section{Descriptors: Elder Abuse; Aging; Prevalence; Depression; Social support.}

\section{Resumen}

Objetivo: Estudio fue caracterizar la prevalencia de violencia contra ancianos residentes en la región metropolitana del municipio de Natal, 
$R N, y$ verificar la relación de este fenómeno con el apoyo social $y$ síntomas depresivos. Método: Estudio descriptivo y transversal, realizado en el período de 24 de octubre de 2016 a 26 de mayo de 2017, con muestra, por conveniencia, de 199 ancianos residentes en la comunidad. Los datos fueron recolectados por medio de: a) Cuestionario Socio Demográfico, b) la Escala de Screening de la Vulnerabilidad al Abuso (VASS-Br, c) la Escala de Depresión Geriátrica (GDS-15), yd) la Escala de Apoyo Social Y LAS). Resultados: Los datos obtenidos fueron categorizados $y$ analizados a través de estadística descriptiva e inferencial. La mayoría de los participantes fueron mujeres $(n=$ 141, 70,9\%) con edad que varía de 60 a 84 años (M: 67,96; DP: \pm 6,45) residentes con cerca de una a ocho personas en el domicilio (M: 4,07, DP: \pm 1,36). La prevalencia de violencia entre los ancianos de la muestra fue del $16 \%(n=31)$. Conclusión: Los hallazgos apuntan a prevalencia de violencia y asociación con síntomas depresivos semejantes a datos ya citados en la literatura. Sin embargo, se resalta que tales datos pueden estar subnotificados, debido a aspectos como el vínculo de proximidad y / o parentesco del agresor con la víctima o las relaciones de dependencia que existan en la relación víctima-agresor.

\section{Descriptores: Maltrato al Anciano;}

Envejecimiento; Prevalencia;

Depresión; Apoyo Social.

\section{Introdução}

O envelhecimento populacional é um fenômeno vivenciado mundialmente. Associado ao envelhecimento humano tem crescido o número de ocorrências de maus-tratos e violências contra a população idosa ${ }^{(1)}$. É notável a imensa quantidade de idosos que tem sido vítima de violência, negligência, maus tratos ou discriminação. Com frequência e gravidade que abrolham exponencialmente, a violência permeia os espaços de convivência social, acontece no âmbito familiar, domiciliar ou em outras instituições sociais, e os direitos e a cidadania de quem por ela é acometido são cruelmente desrespeitados ${ }^{(2)}$.

A violência contra a pessoa idosa pode ser classificada como violência física, psicológica ou emocional dentre as quais se inclui a violência verbal -, sexual, econômica ou financeira, negligência e autonegligência $^{(3)}$. Apesar da violência 
contra idosos ser um fenômeno em ascensão, que ganha visibilidade social e na mídia, ainda se tem encontrado dificuldades no rastreio, na identificação e na prevenção da violência. Dentre os motivos que levam à interdição da informação e dificuldade de denúncia, que dificultam o enfrentamento da violência e que são elencados como principais agentes que geram a subnotificação da violência, destacam-se o grau de proximidade e/ou parentesco do agressor com a vítima ou as relações de dependência afetivoemocional, de cuidado ou financeira, que existem na relação vítima-agressor, por exemplo ${ }^{(4-6)}$.

Aspectos biopsicossociais têm sido associados a este fenômeno. Por exemplo, aspectos da saúde física como ter sofrido quedas ou ser parcial ou totalmente dependente de cuidados e da saúde psíquica - como manifestar sintomas depressivos graves - são apontados como fatores de risco para a ocorrência da violência contra o idoso. Por outro lado, contar com uma rede de apoio social tem sido apontado como fator que previne e/ou protege o idoso contra a ocorrência do fenômeno ${ }^{(2,4)}$.

Diante disso, o presente estudo tem o objetivo de caracterizar a prevalência de violência contra idosos residentes na região metropolitana do município de Natal, $\mathrm{RN}$, e verificar a relação deste fenômeno com o apoio social e sintomas depressivos.

\section{Método}

Estudo descritivo e transversal, realizado no período de 24 de outubro de 2016 a 26 de maio de 2017, com amostra, por conveniência, de 199 idosos residentes na comunidade. Foram incluídos na amostra aqueles idosos com 60 anos ou mais de idade que consentiram, livre e esclarecidamente, em participar da investigação, que possuíam a capacidade de verbalização oral e que apresentavam suas funções mentais preservadas, estando orientados no tempo e no espaço. A coleta dos dados foi realizada individualmente em sala disponibilizada por serviços de atenção a saúde na comunidade em que foram realizados os estudos, dos quais os idosos participantes eram usuários.

O protocolo da pesquisa foi composto pelos seguintes instrumentos: a) Questionário Sócio Demográfico, que inqueriu sobre dados como idade, escolaridade/tempo de estudo, estado civil, religião, além de características clínicas de saúde, entre outros dados; b) a Escala de Screening da 
Vulnerabilidade ao Abuso (VASS-Br), instrumento de 12 itens utilizado para aferição da ocorrência de violência. $\mathrm{O}$ escore total varia de 0 a 12 , sendo indicado que pontuações acima de 3 são indicativas de risco de violência e valores superiores a 7 pontos podem sugerir uma violência já efetivada $\left.{ }^{(7,8)} ; \mathrm{c}\right)$ a Escala de Depressão Geriátrica(GDS15) usada para rastreamento de sintomas de depressão, com escore que varia de 0 a 15 pontos, sendo valores de 6 a 10 pontos indicativos de sintomas depressivos leves a moderados, e valores de 11 em diante indicativos de depressão grave ou severa; e d) a Escala de Apoio Social (EAS), composta por 19 itens respondidos em escala likert de cinco pontos. O escore varia de 19 a 95, considerando que quanto maior $o$ escore, maior a qualidade da rede de apoio.

Os dados obtidos foram categorizados e analisados através de estatística descritiva e inferencial, fazendo-se uso do teste $t$ de Student e a correlação de Pearson. Para efetuar as análises aqui empreendidas, contou-se com o auxílio de softwares informatizados para tratamento estatístico.

O presente projeto atendeu ao que delimita a Declaração de Helsinki e regulamentações nacionais sobre a pesquisa com seres humanos. Utilizouse o Termo de Consentimento Livre e Esclarecido, assegurando sigilo e confidencialidade aos idosos entrevistados. O presente projeto fora submetido à Comissão Nacional de Ética em Pesquisa (CONEP), e fora obtido parecer favorável, conforme explicita o parecer de $n^{\circ} 97.186 / 2012$.

\section{Resultados}

A maioria dos participantes foram mulheres $(\mathrm{n}=141,70,9 \%)$ com idade variando de 60 a 84 anos (M: 67,96; DP: $\pm 6,45)$ residentes com cerca de uma a oito pessoas no domicílio (M: 4,07; DP: $\pm 1,36)$. Os demais dados de caracterização da amostra podem ser observados na tabela 1. Predomina, nesta pesquisa, o perfil de participantes casados $(\mathrm{n}=145,72,9 \%)$, os quais recebem aposentadoria e/ou pensão $(\mathrm{n}=$ $176,88,4 \%$ ).

No que tange aos resultados referentes a VASS-Br, utilizada para aferir risco de violência e/ou violência instaurada, verifica-se que a pontuação na escala variou de 0 a 10 (M: 3,72; DP: $\pm 2,26$ ). A prevalência de violência entre os idosos da amostra investigada foi de $16 \%$ (valor $\geq 7 ; \mathrm{n}=31)$. O escore médio para apoio social, por sua vez, foi de 85,95 (DP: $\pm 11,65$ ), o que sugere uma 
boa percepção sobre a qualidade do apoio que recebe da rede que o assiste. Já para os sintomas depressivos, o escore médio foi de 4,45 (DP: $\pm 2,36$ ). A tabela 2 mostra a correlação entre $o$ escore da VASS-Br com a EAS e o GDS-15.

Tabela 1. Dados Sociodemográficos da População de Idosos Pesquisados

\begin{tabular}{|c|c|c|c|}
\hline Variáveis & & & $\%$ \\
\hline Sove & Feminino & 141 & 70,9 \\
\hline sexo & Masculino & 58 & 29,1 \\
\hline & 60 a 64 & 73 & 36,7 \\
\hline Faixa etária & 65 a 69 & 58 & 29,1 \\
\hline 1 anхa ctanta & 70 a 74 & 27 & 13,6 \\
\hline & 75 a 79 & 25 & 12,6 \\
\hline & 80 a 84 & 16 & 8 \\
\hline Estado Civil & Sim & 145 & 72,9 \\
\hline & Não & 54 & 27,1 \\
\hline Fica só a maior parte do dia? & Sim & 40 & 20,1 \\
\hline & Não & 159 & 79,9 \\
\hline & Nunca estudou & 14 & 7 \\
\hline Escolaridade & $\begin{array}{l}\text { Ensino } \\
\text { fundamental }\end{array}$ & 60 & 30,2 \\
\hline & Ensino Médio & 82 & 41,2 \\
\hline & Ensino superior & 43 & 21,6 \\
\hline Renda própria, pensão ou aposentadoria? & Sim & 176 & 88,4 \\
\hline & Não & 23 & 11,6 \\
\hline Renda familiar & Até $2 \mathrm{SM}$ & 166 & 83,4 \\
\hline & Acima $2 \mathrm{SM}$ & 33 & 16,6 \\
\hline Incapacitado(a) de realizar alguma atividade por motivo de & Sim & 32 & 16,1 \\
\hline saude nos uitımos seis meses! & Não & 168 & 83,9 \\
\hline Sofreu quedas nos últimos seis meses? & Sim & 35 & 17,6 \\
\hline & Não & 164 & 82,4 \\
\hline
\end{tabular}

Legenda: Variáveis: aspecto sociodemográfico investigado; f: frequência absoluta; \%: porcentagem.

No que tange aos resultados referentes a VASS-Br, utilizada para aferir risco de violência e/ou violência instaurada, verifica-se que a pontuação na escala variou de 0 a 10 (M: 3,72; DP: $\pm 2,26)$. A prevalência de violência entre os idosos da amostra investigada foi de $16 \%$ (valor $\geq 7 ; \mathrm{n}=31)$. O escore médio para apoio social, por sua vez, foi de 85,95 (DP: $\pm 11,65$ ), o que sugere uma 
boa percepção sobre a qualidade do apoio que recebe da rede que o assiste. Já para os sintomas depressivos, o escore médio foi de 4,45 (DP: $\pm 2,36$ ). A tabela 2 mostra a correlação entre $o$ escore da VASS-Br com a EAS e o GDS-15.

Tabela 2. Correlação entre Apoio Social e Depressão com VASS-Br

\begin{tabular}{|c|c|c|c|}
\hline \multicolumn{4}{|c|}{ Variáveis } \\
\hline & & GDS-15 & EAS \\
\hline & $\mathrm{r}$ & 0,540 & $-0,138$ \\
\hline VASS-Br & $\mathrm{p}$ & $<0,001 *$ & 0,510 \\
\hline
\end{tabular}

Legenda: r: correlação de Pearson; p: valor da significância estatística do teste Correlação de Pearson; *: $\mathrm{p}$ valor muito significativo $(\mathrm{p}<0,001)$.

Este dado sugere que há correlação forte e positiva entre sintomas depressivos e o risco de violência, dado que sugere que à medida que aumenta os sintomas depressivos a risco aumentado para o idoso vir a ser vítima de violência. Por outro lado, não fora observado tal comportamento entre o apoio social e o risco de violência.

Neste estudo predominou participantes do sexo feminino, o que tem sido associado a maior longevidade destas em relação aos idosos homens, assim como em função destas buscarem com maior assiduidade e frequência serviços de saúde e espaços de convivência para idosos. Destaca-se ainda que, na população investigada, predomina ainda um perfil de idosos que reside em lares multigeracionais e que ficam grande parte do dia acompanhado de filhos e/ou netos, compatível com o perfil apontado na literatura $^{(9)}$.

A prevalência encontrada neste estudo é semelhante as estimativas obtidas em outros estudos epidemiológicos, os quais sugerem prevalências entre $10 \%$ a $21 \%$, em estudos nacionais, e de $15 \%$ a $35 \%$, em estudos internacionais $^{(4)}$.

Estes achados demonstram que o fenômeno da violência se correlaciona com a presença de sintomas depressivos, aproximando-se com os resultados obtidos por Dong, Beck e Simon ${ }^{10}$, os quais averiguaram que os sintomas de depressão estavam associados com maior risco de violência entre os idosos, especialmente para as mulheres. Resultados semelhantes também foram verificados pelo estudo de Dantas, Oliveira e Silveira $^{(11)}$, em que a presença de sintomas depressivos 
demonstrou associação com o aumento no escore da VASS-Br.

Por outro lado, o apoio social não mostrou correlação estatisticamente significativa com à violência. Este resultado destoa de dados encontrados na literatura, que mostraram correlação negativa entre o apoio social percebido e o risco de violência ${ }^{(10)}$.

\section{Conclusão}

Os achados apontam para prevalência de violência e associação com sintomas depressivos semelhantes a dados já citados na literatura. No entanto, ressalta-se que tais dados podem estar subnotificados, devido aos aspectos apontados na literatura, como vínculo de proximidade e/ou parentesco do agressor com a vítima ou as relações de dependência que existam na relação vítima-agressor. A multigeracionalidade do lar, encontrado neste estudo, pode ter impactado na correlação entre a violência e o apoio social.

\section{Referências}

1. Gallione C, Dal Molin A, Cristina FVB, Ferns H, Mattioli M, Suardi B. Screening tools for identification of elder abuse: a systematic review. J Clin Nurs. 2017; 26(15-16):2154-76.
2. Oliveira AAV, Trigueiro DRG, Fernandes MGM, Silva AO. Maustratos a idosos: revisão integrativa da literatura. Rev. bras. enferm. 2013; 66(1):128-33.

3. Correia TMP, Leal MCC, Marques APO, Salgado RAG, Melo HMA. Perfil dos idosos em situação de violência atendidos em serviço de emergência em Recife-PE. Rev. bras. geriatr. gerontol. 2012; 15(3): 529-36.

4. Maia RS, Maia EMC. Prevalence of violence and related aspects: preliminary study of older adults. J Nurs UFPE On Line. 2015; 9(7): 896168.

5. Rodrigues CL, Armond JE, Gorios C. Agressões físicas e sexuais contra idosos notificadas na cidade de São Paulo. Rev. bras. geriatr. gerontol. 2015; 18(4): 755-60.

6. Garbin CAS, Joaquim RC, Rovida TAS, Garbin AJI. Idosos vítimas de maus-tratos: cinco anos de análise documental. Rev. bras. geriatr. gerontol. 2016; 19(1): 87-4.

7. Maia RS, Maia EMC. Adaptação transcultural para o português (Brasil) da Vulnerability to Abuse Screening Scale (VASS) para rastreio da violência contra idosos. Cad. Saúde Pública. 2014; 30(7): 1379-84.

8. Maia RS, Maia EMC. Psychometric evidence of the transcultural adaptation of the Vulnerability Abuse Screening Scale (VASS) for the detection of violence against the elderly. Rev. bras. geriatr. gerontol. 2016; 19(6): 958-69.

9. Ferreira, C. L., Santos, L. M. O., \& Maia, E. M. C. (2012). Resiliência em idosos atendidos na Rede de Atenção Básica de Saúde em município do nordeste brasileiro. Rev. esc. enferm. USP. 2012; 46(2): 328-34. 
10. Dong X, Beck T, Simon MA. The associations of gender, depression and elder mistreatment in a communitydwelling Chinese population: The modifying effect of social support. Arch Gerontol Geriatr. 2010; 50(2):202-08.

11. Dantas RB, Oliveira GL, Silveira AM. Psychometric properties of the Vulnerability to Abuse Screening Scale for screening abuse of older adults. Rev. Saúde Pública. 2017; 51(1): 1-10. 